\title{
TITLE:
}

\section{Radion and holographic brane gravity}

$\operatorname{AUTHOR}(S)$ :

Kanno, S; Soda, J

\section{CITATION:}

Kanno, S ...[et al]. Radion and holographic brane gravity. PHYSICAL REVIEW D 2002, 66(8): 083506.

ISSUE DATE:

2002-10-15

URL:

http://hdl.handle.net/2433/49935

RIGHT:

Copyright 2002 American Physical Society 
PHYSICAL REVIEW D 66, 083506 (2002)

\title{
Radion and holographic brane gravity
}

\author{
Sugumi Kanno* \\ Graduate School of Human and Environmental Studies, Kyoto University, Kyoto 606-8501, Japan \\ Jiro Soda ${ }^{\dagger}$ \\ Department of Fundamental Sciences, FIHS, Kyoto University, Kyoto 606-8501, Japan
}

(Received 8 July 2002; published 16 October 2002)

\begin{abstract}
The low energy effective theory for the Randall-Sundrum two-brane system is investigated with an emphasis on the role of the nonlinear radion in the brane world. The equations of motion in the bulk are solved using a low energy expansion method. This allows us, through the junction conditions, to deduce the effective equations of motion for gravity on the brane. It is shown that the gravity on the brane world is described by a quasi-scalar-tensor theory with a specific coupling function $\omega(\Psi)=3 \Psi / 2(1-\Psi)$ on the positive tension brane and $\omega(\Phi)=-3 \Phi / 2(1+\Phi)$ on the negative tension brane, where $\Psi$ and $\Phi$ are nonlinear realizations of the radion on the positive and negative tension branes, respectively. In contrast with the usual scalar-tensor gravity, the quasi-scalar-tensor gravity couples with two kinds of matter; namely, the matter on both positive and negative tension branes, with different effective gravitational coupling constants. In particular, the radion disguised as the scalar fields $\Psi$ and $\Phi$ couples with the sum of the traces of the energy-momentum tensor on both branes. In the course of the derivation, it is revealed that the radion plays an essential role in converting the nonlocal Einstein gravity with generalized dark radiation to local quasi-scalar-tensor gravity. For completeness, we also derive the effective action for our theory by substituting the bulk solution into the original action. It is also shown that quasi-scalar-tensor gravity works as a hologram at low energy in the sense that the bulk geometry can be reconstructed from the solution of quasi-scalar-tensor gravity.
\end{abstract}

DOI: 10.1103/PhysRevD.66.083506

PACS number(s): 98.80.Cq, 04.50.+h, 98.80.Hw

\section{INTRODUCTION}

Motivated by the recent development of superstring theory, the brane world scenario has been studied intensively. In particular, the warped compactification mechanism proposed by Randall and Sundrum (RS) has given birth to a new picture of the universe [1]. The single brane model (RS2) has been well studied so far because of its simplicity and the absence of a stability problem of the radion mode [2-5]. As for the two-brane model (RS1), Garriga and Tanaka have shown that the gravity on the brane behaves as in BransDicke theory at a linearized level [6]. Thus, the conventional linearized Einstein equations do not hold even on scales large compared with the curvature scale $l$ in the bulk. Charmousis et al. have clearly identified the Brans-Dicke field as the radion mode [7]. Subsequent research has been focused on the role of the radion in the brane world scenario [8-10].

However, the above-mentioned works are restricted to linear theory or to homogeneous cosmological models. It is important to study nonlinear gravity for applications to astrophysical and cosmological problems. Recently, Wiseman has analyzed a special two-brane system with the negative tension brane taken to be in vacuum and has shown that the low energy effective theory becomes a scalar-tensor theory with a specific coupling function [11]. Here, we consider the general case including matter on the negative tension brane and derive the effective equations of motion for this system using a low energy expansion method developed by us [12]

\footnotetext{
*Electronic address: kanno@phys.h.kyoto-u.ac.jp

${ }^{\dagger}$ Electronic address: jiro@phys.h.kyoto-u.ac.jp
}

To further illuminate the role of the radion in the brane world, let us pose the issue in the following way. In our previous paper, we derived the low energy effective equation on the brane as [12] (see also [13])

$$
G_{\nu}^{\mu}=\frac{\kappa^{2}}{l} T_{\nu}^{\mu}-\frac{2}{l} \chi_{\nu}^{\mu}\left(x^{\mu}\right),
$$

where $G^{\mu}{ }_{\nu}, \kappa$, and $T^{\mu}{ }_{\nu}$ denote the four-dimensional Einstein tensor, the five-dimensional gravitational constant, and the energy-momentum tensor on the brane, respectively. Here, the "constant of integration" $\chi_{\mu \nu}(x)$ is transverse and traceless. When we impose maximal symmetry on the spatial part of the brane world, Eq. (1) reduces to the Friedmann equation with dark radiation:

$$
H^{2}=\frac{8 \pi G \rho}{3}+\frac{\mathcal{C}}{a_{0}^{4}}
$$

where $H, a_{0}$, and $\rho$ are, respectively, the Hubble parameter, the scale factor, and the total energy density of each brane, while $\mathcal{C}$ is a constant of integration associated with the mass of a black hole in the bulk. Hence, $\chi_{\mu \nu}(x)$ can be regarded as a generalization of the dark radiation appearing in Eq. (2). The point is that Eq. (1) holds irrespective of the existence of other branes. The effect of the bulk geometry comes into the brane world only through $\chi_{\mu \nu}$.

On the other hand, as we have noted, a scalar-tensor theory emerges in the two-brane system. How can we reconcile these seemingly incompatible pictures? In this paper, we reveal a mechanism to convert the Einstein equations with generalized dark radiation to quasi-scalar-tensor gravity. 
Eventually, it turns out that the radion disentangles the nonlocality in the nonconventional Einstein equations and leads to local quasi-scalar-tensor gravity.

This paper is organized as follows. In Sec. II, our iteration scheme to solve the Einstein equations at low energy is explained. In Sec. III, the background solution is presented. In Sec. IV, we derive the brane effective action from the junction conditions at leading order. We see that the effective theory is described by quasi-scalar-tensor gravity with a specific coupling function. The relation to holography is also discussed. In Sec. V, a systematic method for computing the higher order corrections is discussed. Section VI is devoted to discussion and conclusions. In Appendix A, we explain the physical meaning of our method, especially the relation to the zero mode and Kaluza-Klein modes in linear theory, by using a simple scalar field model. In Appendix B, linearized gravity is analyzed in detail using our method.

\section{LOW ENERGY APPROXIMATION}

\section{A. RS1 model and basic equations}

The model is described by the action

$$
\begin{aligned}
S= & \frac{1}{2 \kappa^{2}} \int d^{5} x \sqrt{-g}\left(\mathcal{R}+\frac{12}{l^{2}}\right)-\sum_{i=A, B} \sigma_{i} \int d^{4} x \sqrt{-g^{i \text { brane }}} \\
& +\sum_{i=A, B} \int d^{4} x \sqrt{-g^{i \text { brane }}} \mathcal{L}_{\text {matter }}^{i}
\end{aligned}
$$

where $\mathcal{R}, g_{\mu \nu}^{i b r a n e}$, and $\kappa^{2}$ are the scalar curvature, the induced metric on the branes, and the gravitational constant in five-dimensions, respectively. We consider an $S_{1} / Z_{2}$ orbifold spacetime with the two branes as the fixed points. In the RS1 model, two flat three-branes are embedded in the fivedimensional asymptotically anti-de Sitter (AdS) bulk with curvature radius $l$ with the brane tensions given by $\sigma_{A}$ $=6 /\left(\kappa^{2} l\right)$ and $\sigma_{B}=-6 /\left(\kappa^{2} l\right)$.

For general nonflat branes, we cannot keep both the two branes straight in the Gaussian normal coordinate system. Hence, we use the following coordinate system to describe the geometry of the brane model:

$$
d s^{2}=e^{2 \phi\left(y, x^{\mu}\right)} d y^{2}+g_{\mu \nu}\left(y, x^{\mu}\right) d x^{\mu} d x^{\nu} .
$$

We place the branes at $y=0$ ( $A$-brane) and $y=l$ ( $B$-brane) in this coordinate system. The proper distance between the two branes with fixed $x$ coordinates can be written as

$$
d(x)=\int_{0}^{l} e^{\phi(y, x)} d y .
$$

Hence, we call $\phi$ the radion (see Fig. 1). In this coordinate system, the five-dimensional Einstein equations become

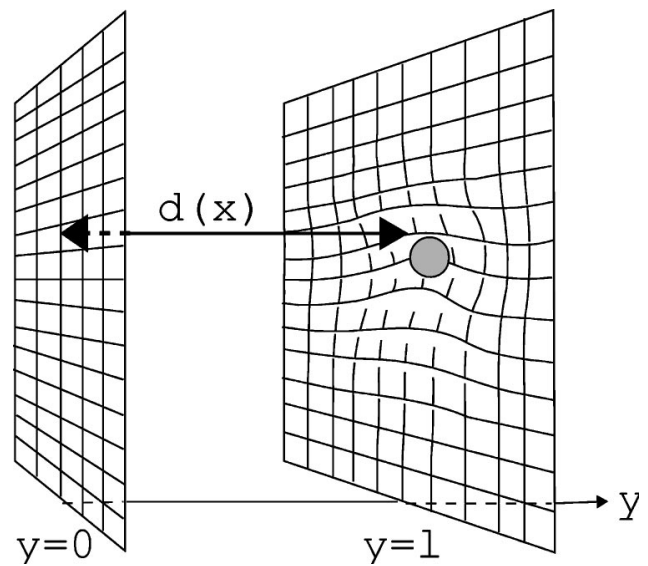

FIG. 1. Radion as a distance between two branes.

$$
\begin{gathered}
e^{-\phi}\left(e^{-\phi} K_{\nu}^{\mu}{ }_{\nu}{ }_{, y}-\left(e^{-\phi} K\right)\left(e^{-\phi} K_{\nu}^{\mu}\right)+R_{\nu}^{\mu}{ }_{\nu}^{(4)}\right. \\
-\nabla^{\mu} \nabla_{\nu} \phi-\nabla^{\mu} \phi \nabla_{\nu} \phi \\
=-\frac{4}{l^{2}} \delta_{\nu}^{\mu}+\kappa^{2}\left(\frac{1}{3} \delta_{\nu}^{\mu} \sigma_{A}+T^{A \mu}{ }_{\nu}-\frac{1}{3} \delta_{\nu}^{\mu} T^{A}\right) e^{-\phi} \delta(y) \\
+\kappa^{2}\left(\frac{1}{3} \delta_{\nu}^{\mu} \sigma_{B}+\widetilde{T}^{B \mu}{ }_{\nu}-\frac{1}{3} \delta_{\nu}^{\mu} \widetilde{T}^{B}\right) e^{-\phi} \delta(y-l), \\
e^{-\phi}\left(e^{-\phi} K\right)_{, y}-\left(e^{-\phi} K^{\alpha \beta}\right)\left(e^{-\phi} K_{\alpha \beta}\right) \\
-\nabla^{\alpha} \nabla_{\alpha} \phi-\nabla^{\alpha} \phi \nabla_{\alpha} \phi \\
=-\frac{4}{l^{2}}-\frac{\kappa^{2}}{3}\left(-4 \sigma_{A}+T^{A}\right) e^{-\phi} \delta(y) \\
-\frac{\kappa^{2}}{3}\left(-4 \sigma_{B}+\widetilde{T}^{B}\right) e^{-\phi} \delta(y-l), \\
\nabla_{\nu}\left(e^{-\phi} K^{\nu}{ }_{\mu}\right)-\nabla_{\mu}\left(e^{-\phi} K\right)=0
\end{gathered}
$$

(4)

where $R^{\mu}{ }_{\nu}$ is the curvature on the brane, $\nabla_{\mu}$ denotes the covariant derivative with respect to the metric $g_{\mu \nu}$, and we introduced the tensor $K_{\mu \nu}=-g_{\mu \nu, y} / 2$ for convenience. One can read off the junction condition from the above equations as

$$
\begin{aligned}
& \left.e^{-\phi}\left[K_{\nu}^{\mu}-\delta_{\nu}^{\mu} K\right]\right|_{y=0}=\frac{\kappa^{2}}{2}\left(-\sigma_{A} \delta_{\nu}^{\mu}+T_{\nu}^{A \mu}\right), \\
& \left.e^{-\phi}\left[K_{\nu}^{\mu}-\delta_{\nu}^{\mu} K\right]\right|_{y=l}=-\frac{\kappa^{2}}{2}\left(-\sigma_{B} \delta_{\nu}^{\mu}+\widetilde{T}_{\nu}^{B \mu}\right),
\end{aligned}
$$

where $K_{\nu}^{\mu}=g^{\mu \alpha} K_{\alpha \nu}$ and the fact that we are considering a $Z_{2}$ symmetric spacetime is used. Decompose the extrinsic curvature into the traceless part and the trace part: 


$$
e^{-\phi} K_{\mu \nu}=\Sigma_{\mu \nu}+\frac{1}{4} g_{\mu \nu} Q, \quad Q=-e^{-\phi} \frac{\partial}{\partial y} \log \sqrt{-g} .
$$

Then, off the brane, we obtain the basic equations

$$
\begin{gathered}
e^{-\phi \Sigma_{\nu, y}^{\mu}-Q \Sigma^{\mu}{ }_{\nu}=}-\left[R_{\nu}^{(4)}-\frac{1}{4} \delta_{\nu}^{\mu} R-\nabla^{\mu} \nabla_{\nu} \phi-\nabla^{\mu} \phi \nabla_{\nu} \phi\right. \\
\left.+\frac{1}{4} \delta_{\nu}^{\mu}\left(\nabla^{\alpha} \nabla_{\alpha} \phi+\nabla^{\alpha} \phi \nabla_{\alpha} \phi\right)\right], \\
\frac{3}{4} Q^{2}-\Sigma_{\beta^{\alpha} \Sigma_{\alpha}^{\beta}}=\left[\begin{array}{c}
(4) \\
R
\end{array}\right]+\frac{12}{l^{2}}, \\
e^{-\phi} Q_{, y}-\frac{1}{4} Q^{2}-\Sigma^{\alpha \beta} \Sigma_{\alpha \beta}=\nabla^{\alpha} \nabla_{\alpha} \phi+\nabla^{\alpha} \phi \nabla_{\alpha} \phi-\frac{4}{l^{2}} \\
\nabla_{\lambda} \Sigma_{\mu}^{\lambda}-\frac{3}{4} \nabla_{\mu} Q=0 .
\end{gathered}
$$

The junction conditions are

$$
\begin{aligned}
& {\left.\left[\Sigma_{\nu}^{\mu}-\frac{3}{4} \delta_{\nu}^{\mu} Q\right]\right|_{y=0}=\frac{\kappa^{2}}{2}\left(-\sigma_{A} \delta_{\nu}^{\mu}+T_{\nu}^{A \mu}\right),} \\
& {\left.\left[\Sigma_{\nu}^{\mu}-\frac{3}{4} \delta_{\nu}^{\mu} Q\right]\right|_{y=l}=-\frac{\kappa^{2}}{2}\left(-\sigma_{B} \delta_{\nu}^{\mu}+\widetilde{T}_{\nu}^{B \mu}\right)}
\end{aligned}
$$

The problem now is separated into two parts. First, we must solve the bulk equations of motion with the Dirichlet boundary condition at the $A$-brane, $g_{\mu \nu}\left(y=0, x^{\mu}\right)=h_{\mu \nu}\left(x^{\mu}\right)$. Then, the junction condition is imposed at each brane. As the junction conditions constrain the induced metrics on both branes, they naturally give rise to the effective equations of motion for gravity on the branes.

\section{B. Low energy expansion scheme}

Unfortunately, it is a formidable task to solve the fivedimensional Einstein equations exactly. However, notice that typically the length scale of the internal space is $l \ll 0.1 \mathrm{~mm}$. On the other hand, the usual astrophysical and cosmological phenomena take place at scales much larger than this scale. Thus we need only the low energy effective theory to analyze a variety of problems, for example, the formation of a black hole, the propagation of gravitational waves, the evolution of cosmological perturbations, and so on. It should be stressed that low energy does not necessarily implies weak gravity on the branes.

Along the normal coordinate $y$, the metric varies with the characteristic length scale $l ; g_{\mu \nu, y} \sim g_{\mu \nu} / l$. Denote the characteristic length scale of the curvature on the brane as $L$. Then we have $R \sim g_{\mu \nu} / L^{2}$. For the reader's reference, let us take $l=1 \mathrm{~mm}$, for example. Then the relations in the RS1 model

$$
\kappa^{2} \sigma_{A}=\frac{6}{l}, \quad \frac{\kappa^{2}}{l}=8 \pi G_{N}
$$

give $\kappa^{2} \sim\left(10^{8} \mathrm{GeV}\right)^{-3}$ and $\left|\sigma_{i}\right| \sim 1 \mathrm{TeV}^{4}$.

In this paper, we will consider the low energy regime in the sense that the energy density of the matter, $\rho_{i}$, on a brane is smaller than the brane tension, i.e., $\rho_{i} /\left|\sigma_{i}\right| \ll 1$. In this regime, a simple dimensional analysis

$$
\frac{\rho_{i}}{\left|\sigma_{i}\right|} \sim \frac{l}{\kappa^{2}\left|\sigma_{i}\right|} \frac{\kappa^{2} \rho_{i}}{l} \sim\left(\frac{l}{L}\right)^{2} \ll 1
$$

implies that the curvature on the brane can be neglected compared with the extrinsic curvature at low energies. Thus, the anti-Newtonian or gradient expansion method used in the cosmological context [14] is applicable to our problem.

Our iteration scheme is to write the metric $g_{\mu \nu}$ as a sum of local tensors built out of the induced metric on the brane, with the number of derivatives increasing with the order of iteration, that is, $O\left((l / L)^{2 n}\right), n=0,1,2, \ldots$ Hence, we seek the metric as a perturbative series

$$
\begin{aligned}
g_{\mu \nu}\left(y, x^{\mu}\right)= & a^{2}(y, x)\left[h_{\mu \nu}\left(x^{\mu}\right)+g_{\mu \nu}^{(1)}\left(y, x^{\mu}\right)\right. \\
& \left.+g_{\mu \nu}^{(2)}\left(y, x^{\mu}\right)+\ldots\right], \\
g_{\mu \nu}^{(n)}\left(y=0, x^{\mu}\right)= & 0, \quad n=1,2,3, \ldots,
\end{aligned}
$$

where the factor $a^{2}(y, x)$ is extracted for a reason explained later and we use the Dirichlet boundary condition $g_{\mu \nu}(y$ $=0, x)=h_{\mu \nu}(x)$ at the $A$-brane. We do not need to know the geometry of the $B$-brane when we focus on the effective equations on the $A$-brane. In other words, from a viewpoint on the $A$-brane, the junction condition at the $B$-brane simply gives the boundary condition for the bulk geometry. Other quantities are also expanded as

$$
\Sigma_{\nu}^{\mu}{ }_{\nu} \Sigma^{(0) \mu}{ }_{\nu}+\Sigma^{(1) \mu}{ }_{\nu}+\Sigma^{(2) \mu}{ }_{\nu}+\cdots .
$$

In Appendix A, we illustrate our method using a simple scalar field example to clarify the relation of the low energy expansion to the zero mode and Kaluza-Klein modes in linearized theory.

\section{BACKGROUND GEOMETRY}

As we can ignore the matter at the lowest order, we obtain a vacuum brane; namely, we have an almost flat brane compared with the curvature scale of the bulk spacetime. At the zeroth order, we can neglect the curvature term. Then we have

$$
\begin{gathered}
e^{-\phi} \Sigma_{\nu, y}^{(0) \mu}-Q^{(0)} \Sigma_{\nu}^{(0) \mu}=0, \\
\frac{3}{4} Q^{(0) 2}-\Sigma_{\beta}^{(0) \alpha} \Sigma_{\alpha}^{(0) \beta}=\frac{12}{l^{2}}, \\
e^{-\phi} Q_{, y}^{(0)}-\frac{1}{4} Q^{(0) 2}-\Sigma^{(0) \alpha \beta} \Sigma_{\alpha \beta}^{(0)}=-\frac{4}{l^{2}},
\end{gathered}
$$




$$
\nabla_{\lambda} \Sigma_{\mu}^{(0) \lambda}-\frac{3}{4} \nabla_{\mu} Q^{(0)}=0
$$

The junction condition is

$$
\begin{aligned}
& {\left.\left[\Sigma_{\nu}^{(0) \mu}-\frac{3}{4} \delta_{\nu}^{\mu} Q^{(0)}\right]\right|_{y=0}=-\frac{\kappa^{2}}{2} \sigma_{A} \delta_{\nu}^{\mu},} \\
& {\left.\left[\Sigma_{\nu}^{(0) \mu}-\frac{3}{4} \delta_{\nu}^{\mu} Q^{(0)}\right]\right|_{y=l}=\frac{\kappa^{2}}{2} \sigma_{B} \delta_{\nu}^{\mu} .}
\end{aligned}
$$

Using Eq. (11), Eq. (23) can be readily integrated,

$$
\Sigma_{\nu}^{(0) \mu}=\frac{C^{\mu}{ }_{\nu}\left(x^{\mu}\right)}{\sqrt{-g}}, \quad C_{\mu}^{\mu}=0
$$

where $C^{\mu}{ }_{\nu}$ is a "constant" of integration. This term is not allowed to exist because of the junction conditions (27) and (28). Thus, it is easy to solve the remaining equations. The result is

$$
\Sigma_{\nu}^{(0) \mu}=0, \quad Q^{(0)}=\frac{4}{l} .
$$

Using the definition

$$
K_{\mu \nu}^{(0)}=-\frac{1}{2} \frac{\partial}{\partial y} g_{\mu \nu}^{(0)}=\frac{1}{l} e^{\phi} g_{\mu \nu}^{(0)},
$$

we get the zeroth order metric as

$$
\begin{aligned}
d s^{2} & =e^{2 \phi(y, x)} d y^{2}+a^{2}(y, x) h_{\mu \nu}\left(x^{\mu}\right) d x^{\mu} d x^{\nu}, \\
a(y, x) & =\exp \left[-\frac{1}{l} \int_{0}^{y} d y e^{\phi(y, x)}\right],
\end{aligned}
$$

where the tensor $h_{\mu \nu}$ is the induced metric on the positive tension brane. Note that the metric derived by Charmousis et al., $e^{\phi}=1+2 f(x) e^{2 y / l} / l$, is consistent with this solution [7]. To proceed further, we take the coordinate system to be $\phi(y, x)=\phi(x)$. Then we have $a(y, x)=\exp \left[-y e^{\phi / l]}\right.$. Although this choice of the coordinate system is generally possible at least locally, there may be a global obstruction. However, as we show below, we can consistently get nontrivial solutions. Moreover, we explicitly demonstrate the validity of our choice at the level of linear theory in Appendix B.

Given the zeroth order solution, junction conditions (27) and (28) lead to the well known relations

$$
\kappa^{2} \sigma_{A}=\frac{6}{l}, \quad \kappa^{2} \sigma_{B}=-\frac{6}{l} .
$$

Note that $\phi(x)$ and $h_{\mu \nu}(x)$ are arbitrary functions of $x$ at zeroth order.

\section{HOLOGRAPHIC QUASI-SCALAR-TENSOR GRAVITY}

\section{A. Bulk geometry}

The next order solution is obtained by taking into account the terms neglected at zeroth order. It is at this order that the effect of matter comes in. At the first order, Eqs. (12)-(15) become

$$
\begin{aligned}
e^{-\phi} \Sigma_{\nu, y}^{(1) \mu}-\frac{4}{l} \Sigma_{\nu}^{(1) \mu}= & -\left[R_{\nu}^{\mu}{ }_{\nu}-\nabla^{\mu} \nabla_{\nu} \phi\right. \\
& \left.-\nabla^{\mu} \phi \nabla_{\nu} \phi\right]_{\text {trace less }}^{(1)}, \\
\frac{6}{l} Q^{(1)}= & {\left[\begin{array}{c}
(4) \\
R
\end{array}\right]^{(1)}, } \\
e^{-\phi} Q_{, y}^{(1)}-\frac{2}{l} Q^{(1)}= & {\left[\nabla^{\alpha} \nabla_{\alpha} \phi+\nabla^{\alpha} \phi \nabla_{\alpha} \phi\right]^{(1)}, } \\
\nabla_{\lambda} \Sigma_{\mu}^{(1) \lambda}-\frac{3}{4} \nabla_{\mu} Q^{(1)}= & 0,
\end{aligned}
$$

where the subscript "traceless" represents the traceless part of the quantity in square brackets. The junction conditions are given by

$$
\begin{aligned}
& {\left.\left[\Sigma_{\nu}^{(1) \mu}-\frac{3}{4} \delta_{\nu}^{\mu} Q^{(1)}\right]\right|_{y=0}=\frac{\kappa^{2}}{2} T_{\nu}^{A \mu},} \\
& {\left.\left[\Sigma_{\nu}^{(1) \mu}-\frac{3}{4} \delta_{\nu}^{\mu} Q^{(1)}\right]\right|_{y=l}=-\frac{\kappa^{2}}{2} \widetilde{T}_{\nu}^{B \mu},}
\end{aligned}
$$

where the superscript (1) represents the order of the gradient expansion. Here, $\left[R^{(4) \mu}{ }_{\nu}^{(1)}\right.$ means the Ricci tensor of $a^{2} h_{\mu \nu}$. Note that now $a=\exp \left[-y e^{\phi} / l\right]$. It is convenient to introduce the Ricci tensor of $h_{\mu \nu}$, denoted by $R_{\nu}^{\mu}(h)$, and express $\left[R_{\nu}^{(4) \mu}\right]^{(1)}$ in terms of $R_{\nu}^{\mu}$ and $\phi$;

$$
\begin{aligned}
{\left[R_{\nu}^{(4)}(g)\right]^{(1)}=} & \frac{1}{a^{2}}\left[R_{\nu}^{\mu}(h)+2 \frac{y}{l} e^{\phi}\left(\phi_{\mid \nu}^{\mid \mu}+\phi^{\mid \mu} \phi_{\mid \nu}\right)\right. \\
& +\delta_{\nu}^{\mu} \frac{y}{l} e^{\phi}\left(\phi_{\mid \alpha}^{\mid \alpha}+\phi^{\mid \alpha} \phi_{\mid \alpha}\right)+2 \frac{y^{2}}{l^{2}} e^{2 \phi} \phi^{\mid \mu} \phi_{\mid \nu} \\
& \left.-2 \delta_{\nu}^{\mu} \frac{y^{2}}{l^{2}} e^{2 \phi} \phi^{\mid \alpha} \phi_{\mid \alpha}\right]
\end{aligned}
$$

where | denotes the covariant derivative with respect to $h_{\mu \nu}$. Similarly, it is convenient to express the second derivatives of $\phi$ as

$$
\left[\nabla^{\mu} \nabla_{\nu} \phi\right]^{(1)}=\frac{1}{a^{2}}\left[\phi_{\mid \nu}^{\mid \mu}+2 \frac{y}{l} e^{\phi} \phi^{\mid \mu} \phi_{\mid \nu}-\frac{y}{l} e^{\phi} \delta_{\nu}^{\mu} \phi^{\mid \alpha} \phi_{\mid \alpha}\right] .
$$

Substituting the trace of Eq. (40) into the right-hand side of Eq. (35), we obtain 


$$
Q^{(1)}=\frac{l}{a^{2}}\left[\frac{1}{6} R(h)+\frac{y e^{\phi}}{l}\left(\phi_{\mid \alpha}^{\mid \alpha}+\phi^{\mid \alpha} \phi_{\mid \alpha}\right)-\frac{y^{2} e^{2 \phi}}{l^{2}} \phi^{\mid \alpha} \phi_{\mid \alpha}\right] .
$$

Note that Eq. (36) is trivially satisfied now. Hereafter, we omit the argument of the curvature for simplicity. Substituting Eqs. (40) and (41) into Eq. (34) and integrating it, we obtain the traceless part of the extrinsic curvature as

$$
\begin{aligned}
\Sigma_{\nu}^{(1) \mu}= & \frac{l}{a^{2}}\left[\frac{1}{2}\left(R_{\nu}^{\mu}-\frac{1}{4} \delta_{\nu}^{\mu} R\right)+\frac{y e^{\phi}}{l}\left(\phi_{\mid \nu}^{\mid \mu}-\frac{1}{4} \delta_{\nu}^{\mu} \phi_{\mid \alpha}^{\mid \alpha}\right)\right. \\
& \left.+\left(\frac{y^{2} e^{2 \phi}}{l^{2}}+\frac{y e^{\phi}}{l}\right)\left(\phi^{\mid \mu} \phi_{\mid \nu}-\frac{1}{4} \delta_{\nu}^{\mu} \phi^{\mid \alpha} \phi_{\mid \alpha}\right)\right] \\
& +\frac{\chi_{\nu}^{\mu}(x)}{a^{4}}
\end{aligned}
$$

where $\chi_{\nu}^{\mu}$ is an integration constant with the property $\chi^{\mu}{ }_{\mu}$ $=0$. And $\chi^{\mu}{ }_{\nu}$ must be transverse, $\chi^{\mu}{ }_{\nu \mid \mu}=0$, in order to satisfy Eq. (37). The definition (11) gives

$$
-\frac{1}{2} e^{-\phi_{g}(0) \alpha \mu} \frac{\partial}{\partial y} g_{\alpha \nu}^{(1)}=\Sigma_{\nu}^{(1) \mu}+\frac{1}{4} \delta_{\nu}^{\mu} Q^{(1)} .
$$

Integrating Eq. (44), we obtain the metric in the bulk:

$$
\begin{aligned}
g_{\mu \nu}^{(1)}= & -\frac{l^{2}}{2}\left(\frac{1}{a^{2}}-1\right)\left(R_{\mu \nu}-\frac{1}{6} h_{\mu \nu} R\right) \\
& +\frac{l^{2}}{2}\left(\frac{1}{a^{2}}-1-\frac{2 y e^{\phi}}{l} \frac{1}{a^{2}}\right)\left(\phi_{\mid \mu \nu}+\frac{1}{2} h_{\mu \nu} \phi^{\mid \alpha} \phi_{\mid \alpha}\right) \\
& -\frac{y^{2} e^{2 \phi}}{a^{2}}\left(\phi_{\mid \mu} \phi_{\mid \nu}-\frac{1}{2} h_{\mu \nu} \phi^{\mid \alpha} \phi_{\mid \alpha}\right)-\frac{l}{2}\left(\frac{1}{a^{4}}-1\right) \chi_{\mu \nu},
\end{aligned}
$$

where we have imposed the boundary condition $g_{\mu \nu}^{(1)}(y$ $\left.=0, x^{\mu}\right)=0$. From these results, one can calculate the Weyl tensor as

$$
C_{y \mu y \nu}=\frac{2 \chi_{\mu \nu}}{l a^{4}} .
$$

Hence, the term $\chi_{\mu \nu}$ is essentially the Weyl tensor at this order. Note that we have obtained the bulk metric in terms of $\phi(x), h_{\mu \nu}(x)$, and $\chi_{\mu \nu}(x)$.

\section{B. Quasi-scalar-tensor gravity}

We shall deduce the equations for $\phi(x), h_{\mu \nu}(x)$, and $\chi_{\mu \nu}(x)$ from the junction conditions. Using Eqs. (42) and (43), one gets the junction conditions. The junction condition at the $A$-brane is written as

$$
\frac{l}{2} G_{\nu}^{\mu}(h)+\chi_{\nu}^{\mu}=\frac{\kappa^{2}}{2} T_{\nu}^{A \mu} .
$$

This equation is nothing but the Einstein equation with generalized dark radiation $\chi_{\mu \nu}$. It should be noted that $\chi_{\mu \nu}$ is undetermined at this level, exhibiting the nonlocal nature of Eq. (47).

The junction condition at the $B$-brane is given by

$$
\begin{gathered}
\frac{l}{2 \Omega^{2}} G_{\nu}^{\mu}+\frac{l e^{\phi}}{\Omega^{2}}\left(\phi_{\mid \nu}^{\mid \mu}-\delta_{\nu}^{\mu} \phi_{\mid \alpha}^{\mid \alpha}+\phi^{\mid \mu} \phi_{\mid \nu}-\delta_{\nu}^{\mu} \phi^{\mid \alpha} \phi_{\mid \alpha}\right) \\
+\frac{l e^{2 \phi}}{\Omega^{2}}\left(\phi^{\mid \mu} \phi_{\mid \nu}+\frac{1}{2} \delta_{\nu}^{\mu} \phi^{\mid \alpha} \phi_{\mid \alpha}\right)+\frac{\chi^{\mu}{ }_{\nu}}{\Omega^{4}}=-\frac{\kappa^{2}}{2 \Omega^{2}} T^{B \mu}{ }_{\nu},
\end{gathered}
$$

where $\Omega(x)=a(y=l, x)=\exp \left[-e^{\phi}\right]$. Here, the index of $T^{B \mu}{ }_{\nu}$ is the energy-momentum tensor with the index raised by the induced metric $h_{\mu \nu}$ on the $A$-brane, while $\widetilde{T}^{B \mu}{ }_{\nu}$ is the one raised by the induced metric on the $B$-brane. At the present order, we have the following relations:

$$
T_{\mu \nu}^{B}=\widetilde{T}_{\mu \nu}^{B}, \quad T_{\nu}^{B \mu}=\Omega^{2} \widetilde{T}_{\nu}^{B \mu} .
$$

To reveal the role of the radion field, we must write Eq. (48) using the induced metric on the $B$-brane, $g_{\mu \nu}^{B \text { brane }}=\Omega^{2}\left(h_{\mu \nu}\right.$ $\left.+g_{\mu \nu}^{(1)}\right) \equiv f_{\mu \nu}+\Omega^{2} g_{\mu \nu}^{(1)}$. At this order, the Ricci tensor $R_{\nu}^{\mu}$ of the induced metric on the $B$-brane is equal to that of $f_{\mu \nu}$. Using this fact, we rewrite Eq. (48) to obtain the effective equations on the $B$-brane:

$$
\frac{l}{2} G_{\nu}^{\mu}(f)+\frac{\chi_{\nu}^{\mu}{ }_{\nu}}{\Omega^{4}}=-\frac{\kappa^{2}}{2} \widetilde{T}^{B \mu}{ }_{\nu} .
$$

Again, we have the nonconventional (nonlocal) Einstein equations, as in the case of the $A$-brane.

Although Eqs. (47) and (50) are nonlocal individually, with undetermined $\chi_{\mu \nu}$, one can combine the two equations to reduce them to local equations for each brane. This happens to be possible since $\chi_{\mu \nu}$ appears only algebraically; one can easily eliminate $\chi_{\mu \nu}$ from Eqs. (47) and (48). Defining a new field $\Psi=1-\Omega^{2}$, we find

$$
\begin{aligned}
G_{\nu}^{\mu}(h)= & \frac{\kappa^{2}}{l \Psi} T_{\nu}^{A \mu}+\frac{\kappa^{2}(1-\Psi)}{l \Psi} T_{\nu}^{B \mu}+\frac{1}{\Psi}\left(\Psi_{\mid \nu}^{\mid \mu}\right. \\
& \left.-\delta_{\nu}^{\mu} \Psi_{\mid \alpha}^{\mid \alpha}\right)+\frac{\omega(\Psi)}{\Psi^{2}}\left(\Psi^{\mid \mu} \Psi_{\mid \nu}-\frac{1}{2} \delta_{\nu}^{\mu} \Psi^{\mid \alpha} \Psi_{\mid \alpha}\right),
\end{aligned}
$$

where the coupling function $\omega(\Psi)$ takes the following form:

$$
\omega(\Psi)=\frac{3}{2} \frac{\Psi}{1-\Psi} .
$$

We can also determine $\chi_{\nu}^{\mu}$ by eliminating $G_{\nu}^{\mu}$ from Eqs. (47) and (48). Then, 


$$
\begin{aligned}
\chi_{\nu}^{\mu}= & -\frac{\kappa^{2}(1-\Psi)}{2 \Psi}\left(T_{\nu}^{A \mu}+T_{\nu}^{B \mu}\right)-\frac{l}{2 \Psi}\left[\left(\Psi_{\mid \nu}^{\mid \mu}-\delta_{\nu}^{\mu} \Psi_{\mid \alpha}^{\mid \alpha}\right)\right. \\
& \left.+\frac{\omega(\Psi)}{\Psi}\left(\Psi^{\mid \mu} \Psi_{\mid \nu}-\frac{1}{2} \delta_{\nu}^{\mu} \Psi^{\mid \alpha} \Psi_{\mid \alpha}\right)\right] .
\end{aligned}
$$

The condition $\chi_{\mu}^{\mu}=0$ gives rise to the field equation for $\Psi$ :

$$
\square \Psi=\frac{\kappa^{2}}{l} \frac{T^{A}+T^{B}}{2 \omega+3}-\frac{1}{2 \omega+3} \frac{d \omega}{d \Psi} \Psi^{\mid \mu} \Psi_{\mid \mu},
$$

where we have used the explicit form of $\omega(\Psi)$. This equation tells us that the trace part of the energy-momentum tensor determines the radion field and hence the relative bending of the brane, and $\chi_{\mu \nu}$ is determined by the traceless part of the right-hand side of Eq. (53). Remarkably, $\chi_{\mu \nu}$ is now a secondary entity.

Equations (51) and (54) are the basic equations to be used in cosmological or astrophysical contexts when the characteristic energy density is less than $\left|\sigma_{i}\right|$. Notice that the conservation law with respect to the metric $h_{\mu \nu}$ reads

$$
T_{\nu \mid \mu}^{A \mu}=0, \quad T_{\nu \mid \mu}^{B \mu}=\frac{\Psi_{\mid \mu}}{1-\Psi} T_{\nu}^{B \mu}-\frac{1}{2} \frac{\Psi_{\mid \nu}}{1-\Psi} T^{B} .
$$

In contrast to the usual scalar-tensor gravity, this theory couples with two kinds of matter, namely, the matter on both positive and negative tension branes, with different effective gravitational coupling constants. For this reason, we call this theory quasi-scalar-tensor gravity. Thus, the (nonlocal) Einstein equation (47) with generalized dark radiation has transformed into the (local) quasi-scalar-tensor gravity (51) with the coupling function $\omega(\Psi)$.

\section{Effective action}

Let us consider an effective action for $h_{\mu \nu}(x)$ and $\phi(x)$. If one wants to calculate the quantum fluctuations in the inflationary scenario, for example, one needs the action to determine their magnitude. The action has to be derived from the original five-dimensional action by substituting the solution of the equations of motion in the bulk and integrating out over the bulk coordinate. We shall start with the following action:

$$
\begin{aligned}
S= & \frac{1}{2 \kappa^{2}} \int d^{5} x \sqrt{-g}\left[\mathcal{R}+\frac{12}{l^{2}}\right]+\frac{2}{\kappa^{2}} \int d^{4} x \sqrt{-h} Q^{A} \\
& -\frac{2}{\kappa^{2}} \int d^{4} x \sqrt{-f} Q^{B}-\frac{6}{\kappa^{2} l} \int d^{4} x \sqrt{-h}+\frac{6}{\kappa^{2} l} \int d^{4} x \sqrt{-f} \\
& +\int d^{4} x \sqrt{-h} \mathcal{L}^{A}+\int d^{4} x \sqrt{-f} \mathcal{L}^{B},
\end{aligned}
$$

where we have taken into account the boundary term, the so-called Gibbons-Hawking term, instead of introducing delta-function singularities in the curvature. The factor 2 in the Gibbons-Hawking term comes from the $Z_{2}$ symmetry of this spacetime. As we substitute the solution of the bulk equations of motion, we can use the equation $\mathcal{R}=-20 / l^{2}$ which holds in the bulk. It should be stressed that the bulk metric is solved without using junction conditions and is expressed in terms of $\phi, h_{\mu \nu}$, and $\chi_{\mu \nu}$. That is why we can get the effective action on the brane by simple substitution of the solution. Now, up to first order, we obtain

$$
\begin{aligned}
S= & -\frac{8}{\kappa^{2} l^{2}} \int d^{4} x \sqrt{-h} \int_{0}^{l e^{\phi}} d z a^{4}\left[1+\frac{1}{2} h^{\mu \nu} g_{\mu \nu}^{(1)}\right]+\frac{2}{\kappa^{2}} \int d^{4} x \sqrt{-h}\left[\frac{4}{l}+\frac{l}{6} R\right]-\frac{2}{\kappa^{2}} \int d^{4} x \sqrt{-h} \Omega^{4}\left[1+\frac{1}{2} h^{\mu \nu} g_{\mu \nu}^{(1)}\right] \\
& \times\left[\frac{4}{l}+\frac{l}{6 \Omega^{2}} R+\frac{l e^{\phi}}{\Omega^{2}}\left(\square \phi+\phi^{\mid \alpha} \phi_{\mid \alpha}\right)-\frac{l e^{2 \phi}}{\Omega^{2}} \phi^{\mid \alpha} \phi_{\mid \alpha}\right]-\frac{6}{\kappa^{2} l} \int d^{4} x \sqrt{-h}+\frac{6}{\kappa^{2} l} \int d^{4} x \sqrt{-h} \Omega^{4}\left[1+\frac{1}{2} h^{\mu \nu} g_{\mu \nu}^{(1)}\right] \\
& +\int d^{4} x \sqrt{-h} \mathcal{L}^{A}+\int d^{4} x \sqrt{-h} \Omega^{4} \mathcal{L}^{B} .
\end{aligned}
$$

Using Eq. (45) and the definition $\Psi=1-\Omega^{2}$, we finally have the action:

$$
\begin{aligned}
S= & \frac{l}{2 \kappa^{2}} \int d^{4} x \sqrt{-h}\left[\Psi R-\frac{\omega(\Psi)}{\Psi} \Psi^{\mid \alpha} \Psi_{\mid \alpha}\right] \\
& +\int d^{4} x \sqrt{-h} \mathcal{L}^{A}+\int d^{4} x \sqrt{-h}(1-\Psi)^{2} \mathcal{L}^{B} .
\end{aligned}
$$

This is a complete derivation of the action with the correct normalization, which is important for quantization of the theory.

Here, it should be noted that $\chi_{\nu}^{\mu}$, which appeared in $g_{\mu \nu}^{(1)}$, is a nonlocal quantity. In fact, eliminating $\Psi$ from Eq. (53) by solving Eq. (54) yields a nonlocal expression for $\chi_{\nu}^{\mu}$. If we substitute this nonlocal expression into Eq. (47), we obtain a nonlocal theory. Conversely, one can see that introducing the radion disentangles the nonlocality in the nonconventional Einstein equation (47) and yields the quasi-scalartensor gravity given by Eqs. (51) and (54). This important point is more transparent in the derivation of the effective 
action. Indeed, the nonlocal part $\chi_{\nu}^{\mu}$ disappears because of the traceless nature, $\chi_{\mu}^{\mu}=0$. A similar mechanism is discussed by Gen and Sasaki [8] in the context of linear theory.

\section{Holographic brane gravity}

We have obtained four-dimensional quasi-scalar-tensor gravity from the five-dimensional action. The bulk metric corresponding to the four-dimensional effective theory is given by

$$
g_{\mu \nu}=(1-\Psi)^{y / l}\left[h_{\mu \nu}(x)+g_{\mu \nu}^{(1)}\left(h_{\mu \nu}, \Psi, T_{\mu \nu}^{A}, T_{\mu \nu}^{B}, y\right)\right],
$$

where $\chi_{\mu \nu}$ in Eq. (45) is eliminated by using Eq. (53). Here, the $y$ dependence of $g_{\mu \nu}^{(1)}$ is explicitly known. Thus the bulk metric is completely determined by the energy-momentum tensors on the two branes, the radion, and the induced metric on the A-brane. Therefore, once the four-dimensional solution of quasi-scalar-tensor gravity is given, one can reconstruct the bulk geometry from these data. Quasi-scalar-tensor gravity works as a hologram at low energy. In this sense, one can call the quasi-scalar-tensor gravity the holographic brane gravity. Equation (59) gives a holographic picture of the brane world. Recalling that the radion specifies the position of the second brane, the radion can be interpreted as a kind of "phase" in the holographic picture of the brane world.

\section{E. Effective theory on $B$-brane}

For completeness, we shall derive the effective equations of motion on the $B$-brane. To do so, let us simply reverse the role of the $A$-brane and that of the $B$-brane. Substituting $h_{\mu \nu}=\Omega^{-2} f_{\mu \nu}$ into the junction conditions yields

$$
\frac{l}{2} G_{\nu}^{\mu}(f)+\frac{\chi_{\nu}^{\mu}}{\Omega^{4}}=-\frac{\kappa^{2}}{2} T_{\nu}^{B \mu}
$$

and

$$
\begin{aligned}
& \frac{l \Omega^{2}}{2} G_{\nu}^{\mu}+l \Omega^{2}\left((\log \Omega)_{; \nu}^{; \mu}-\delta_{\nu}^{\mu}(\log \Omega)_{; \alpha}^{; \alpha}\right. \\
& \left.+(\log \Omega)^{; \mu}(\log \Omega)_{; \nu}+\frac{1}{2} \delta_{\nu}^{\mu}(\log \Omega)^{; \alpha}(\log \Omega)_{; \alpha}\right)+\chi_{\nu}^{\mu} \\
& =\frac{\kappa^{2} \Omega^{2}}{2} T_{\nu}^{A \mu}
\end{aligned}
$$

where; denotes the covariant derivative with respect to the metric $f_{\mu \nu}$. Thus, defining $\Phi=\Omega^{-2}-1$, we obtain the effective equation on the $B$-brane:

$$
\begin{aligned}
G_{\nu}^{\mu}(f)= & \frac{\kappa^{2}}{l \Phi} T_{\nu}^{B \mu}+\frac{\kappa^{2}(1+\Phi)}{l \Phi} T_{\nu}^{A \mu}{ }_{\nu}+\frac{1}{\Phi}\left(\Phi_{; \nu}^{; \mu}-\delta_{\nu}^{\mu} \Phi_{; \alpha}^{; \alpha}\right) \\
& +\frac{\omega(\Phi)}{\Phi^{2}}\left(\Phi^{; \mu} \Phi_{; \nu}-\frac{1}{2} \delta_{\nu}^{\mu} \Phi^{; \alpha} \Phi_{; \alpha}\right),
\end{aligned}
$$

where

$$
\omega(\Phi)=-\frac{3}{2} \frac{\Phi}{1+\Phi} .
$$

The equation of motion for the radion becomes

$$
\Phi=\frac{\kappa^{2}}{l} \frac{T^{A}+T^{B}}{2 \omega+3}-\frac{1}{2 \omega+3} \frac{d \omega}{d \Phi} \Phi^{; \mu} \Phi_{; \mu} .
$$

Thus, we have shown that the gravity on the negative tension brane is described by quasi-scalar-tensor gravity with the coupling function $\omega(\Phi)=-3 \Phi / 2(1+\Phi)$.

It should be noted that the dynamics on the two branes are not independent. We know the gravity on the $B$-brane once we know that on the $A$-brane, and vice versa. The transformation rules are

$$
\begin{aligned}
\Phi & =\frac{\Psi}{1-\Psi}, \\
g_{\mu \nu}^{B \text { brane }} & =(1-\Psi)\left[h_{\mu \nu}+g_{\mu \nu}^{(1)}\left(h_{\mu \nu}, \Psi, T_{\mu \nu}^{A}, T_{\mu \nu}^{B}, y=l\right)\right] .
\end{aligned}
$$

This relation is useful when we consider concrete applications.

\section{KALUZA-KLEIN CORRECTIONS}

As explained in Appendix A, our analysis so far to first order in the gradient expansion corresponds to the zero mode truncation in the language of a linearized theory. Although it is obscure to use the words "Kaluza-Klein corrections" in a nonlinear theory, we shall call their nonlinear counterpart simply Kaluza-Klein corrections in this paper.

In principle, we can continue our analysis up to a desired order using the following recursive formulas:

$$
\begin{aligned}
\Sigma_{\nu}^{(n) \mu}= & -\frac{1}{a^{4}} \int d y a^{4}\left\{\left[\begin{array}{c}
(4) \\
R_{\nu}^{\mu}
\end{array}-\nabla^{\mu} \nabla_{\nu} \phi-\nabla^{\mu} \phi \nabla_{\nu} \phi\right]_{\text {traceless part }}^{(n)}\right. \\
& \left.-\sum_{p=1}^{n-1} Q^{(p)} \Sigma_{\nu}^{(n-p) \mu}\right\}, \\
Q^{(n)}= & \frac{l}{6} \sum_{p=1}^{n-1}\left[-\frac{3}{4} Q^{(p)} Q^{(n-p)}+\Sigma_{\beta}^{(p) \alpha} \Sigma_{\alpha}^{(n-p) \beta}+\left[\begin{array}{c}
(4) \\
R
\end{array}\right]^{(n)}\right],
\end{aligned}
$$

$$
\begin{aligned}
Q_{, y}^{(n)}-\frac{2}{l} Q^{(n)}= & \sum_{p=1}^{n-1}\left\{\frac{1}{4} Q^{(p)} Q^{(n-p)}+\Sigma^{(p) \alpha \beta} \Sigma_{\alpha \beta}^{(n-p)}\right\} \\
& +\left[\nabla^{\alpha} \nabla_{\alpha} \phi+\nabla^{\alpha} \phi \nabla_{\alpha} \phi\right]^{(n)}, \\
\Sigma_{\mu}^{(n) \lambda} \mid \lambda- & -\frac{3}{4} Q_{\mid \mu}^{(n)}+\sum_{p=1}^{n-1}\left\{\Gamma_{\lambda \alpha}^{(p) \alpha} \Sigma^{(n-p) \lambda}{ }_{\mu}\right. \\
- & \left.\Gamma_{\alpha \mu}^{(p) \lambda} \Sigma_{\lambda}^{(n-p) \alpha}{ }_{\lambda}\right\}=0 .
\end{aligned}
$$


These equations give the solution as an infinite sum. The existence of an infinite series is a manifestation of the nonlocality of the brane model [15].

To get the effective equations of motion with second order corrections using the above formula is straightforward. However, carrying out the calculation is laborious and the resultant expression is too long to write down. As for the linear theory, we will obtain the explicit effective equations of motion with Kaluza-Klein corrections in Appendix B. Here, we will only sketch how the Kaluza-Klein corrections appear using an easy method.
Although we need the explicit $y$ dependence of the bulk to obtain the action, as long as we are interested only in the effective equations on the brane, we do not have to solve the bulk explicitly. The reason is as follows. We can write down the nonlocal Einstein equations corresponding to Eqs. (47) and (50) without knowing the bulk geometry. Then, since we know how the nonlocal term, i.e., the generalized dark radiation term, behaves in the bulk, we may eliminate it just as in the first order case.

The nonlocal Einstein equations on the branes are [12]

$$
\begin{aligned}
& G_{\mu \nu}^{(4)}(h)=-\frac{2}{l}\left(\chi_{\mu \nu}+t_{\mu \nu}\right)+\frac{\kappa^{2}}{l} T_{\mu \nu}^{A}-\frac{l^{2}}{2} \mathcal{S}_{\mu \nu}-\frac{l^{2}}{12}\left(R R_{\mu \nu}-\frac{1}{2} h_{\mu \nu} R^{2}+\frac{3}{4} h_{\mu \nu} R_{\beta}^{\alpha} R_{\alpha}^{\beta}\right)+\frac{l}{2}\left[\chi_{\mu \mid \nu \alpha}^{\alpha}+\chi_{\nu \mid \mu \alpha}^{\alpha}-\chi_{\mu \nu \mid \alpha} \mid \alpha\right] \\
& -l \chi_{\mu \alpha} R_{\nu}^{\alpha}+\frac{l}{6} R \chi_{\mu \nu}-\frac{1}{4} h_{\mu \nu} \chi_{\beta}^{\alpha} \chi_{\alpha}^{\beta} \text {, } \\
& G_{\mu \nu}^{(4)}\left(g^{B \text { brane }}\right)=-\frac{2}{l \Omega^{4}}\left(\chi_{\mu \nu}+t_{\mu \nu}\right)-\frac{\kappa^{2}}{l} T_{\mu \nu}^{B}-l^{2}\left(1+\frac{\Omega^{2}}{2}\right) \mathcal{S}_{\mu \nu}-\frac{l^{2}}{12} \Omega^{2}\left(R R_{\mu \nu}-\frac{3}{2} g_{\mu \nu}^{B \text { brane }} R_{\beta}^{\alpha} R_{\alpha}^{\beta}\right)+\frac{l}{4}\left(1+\frac{1}{\Omega^{4}}\right)\left[\chi^{\alpha}{ }_{\mu ; \nu \alpha}\right. \\
& \left.+\chi_{\nu ; \mu \alpha}^{\alpha}-\chi_{\mu \nu ; \alpha}^{; \alpha}\right]-\frac{l}{2}\left(1+\frac{1}{\Omega^{4}}\right)\left(\chi_{\mu \alpha} R_{\nu}^{\alpha}-\frac{1}{4} g_{\mu \nu}^{B \text { brane }} \chi_{\alpha \beta} R^{\alpha \beta}\right)+\frac{l}{6 \Omega^{4}} R \chi_{\mu \nu}-\frac{3}{4} g_{\mu \nu}^{B \text { brane }}\left[\frac{l^{2}}{4}\left(R_{\beta}^{\alpha} R_{\alpha}^{\beta}-\frac{2}{9} R^{2}\right)\right. \\
& \left.+\frac{l}{2 \Omega^{4}} \chi_{\beta}^{\alpha} R_{\alpha}^{\beta}-\frac{l}{6} \chi_{\beta}^{\alpha} R_{\alpha}^{\beta}+\frac{1}{3 \Omega^{8}} \chi_{\beta}^{\alpha} \chi_{\alpha}^{\beta}\right] \text {, }
\end{aligned}
$$

where $t_{\mu \nu}$ is an integration constant at second order and we have defined the quantity

$$
\begin{aligned}
\mathcal{S}_{\nu}^{\mu}= & R_{\alpha}^{\mu} R_{\nu}^{\alpha}-\frac{1}{3} R R_{\nu}^{\mu}-\frac{1}{4} \delta_{\nu}^{\mu}\left(R_{\beta}^{\alpha} R_{\alpha}^{\beta}-\frac{1}{3} R^{2}\right) \\
& -\frac{1}{2}\left(R_{\mid \nu \alpha}^{\alpha \mu}+R_{\nu}^{\alpha}{ }_{\nu}{ }_{\mid \alpha}-\frac{2}{3} R_{\mid \nu}^{\mid \mu}-\square R_{\nu}^{\mu}+\frac{1}{6} \delta_{\nu}^{\mu} \square R\right) .
\end{aligned}
$$

Here, ; represents the covariant derivative with respect to $f_{\mu \nu}$ and all the curvatures in Eq. (72) are calculated from $g_{\mu \nu}^{B \text { brane }}$. What we should do is to eliminate $t_{\mu \nu}$ from Eqs. (71) and (72) and substitute the relation $g_{\mu \nu}^{B \text { brane }}=\Omega^{2}\left[h_{\mu \nu}\right.$ $\left.+g_{\mu \nu}^{(1)}\right]$ into the resulting equation. Then we obtain a higher derivative but local theory on the brane.

Noticeably, the same is true for all higher order corrections. Thus, one can infer that the radion disentangles the nonlocality in the system to all orders at the expense of introducing higher derivative terms.

\section{CONCLUSION}

We have developed a method to deduce a low energy effective theory for a two-brane system. The fivedimensional equations of motion in the bulk are solved using a low energy expansion method. This allows us, through the junction conditions, to deduce the effective equations of motion for gravity on the brane. As a result, we have shown that gravity on the brane world is described by a quasi-scalartensor theory with a specific coupling function $\omega(\Psi)$ $=3 \Psi / 2(1-\Psi)$ on the positive tension brane and $\omega(\Phi)=$ $-3 \Phi / 2(1+\Phi)$ on the negative tension brane, where $\Psi$ and $\Phi$ are Brans-Dicke-like scalars on the positive and negative tension branes, respectively. In contrast to the usual scalartensor theory, the quasi-scalar-tensor theory couples with matter on both branes but with different effective gravitational coupling constants. In particular, the radion disguised as the scalar fields $\Psi$ and $\Phi$ couples with the sum of the traces of the energy-momentum tensors on both branes. Moreover, we have derived the effective action by substituting the solution of the bulk equations of motion into the original action. This direct method determines the normalization of the effective action, which is indispensable for quantizing the theory.

In the process of derivation of the effective equations of motion, we have clarified how quasi-scalar-tensor gravity emerges from Einstein's theory with a generalized dark radiation term described by $\chi_{\mu \nu}$. A brane can feel the nonlocal effect of the bulk geometry only through $\chi_{\mu \nu}$, irrespective of the existence of another brane. This is the picture given to us by Einstein equations with generalized dark radiation. Then, what is the role of the radion? In order to make the connection between the radion and $\chi_{\mu \nu}$, we have to know the bulk 
geometry. In the case of a single brane, $\chi_{\mu \nu}$ is determined by the boundary conditions at the Cauchy horizon. If we require that the geometry is asymptotically anti-de Sitter there, then $\chi_{\mu \nu}$ must vanish [16]. In the two-brane case, we have no asymptotic region; instead we have the second brane in the bulk. The radion determines the location of the second brane where the junction conditions are imposed. The junction conditions give $\chi_{\mu \nu}$ as a function of the energy-momentum tensor and the radion. The resultant equation is nothing but the holographic quasi-scalar-tensor gravity. Thus, the difference between the Einstein equations with generalized dark radiation and quasi-scalar-tensor gravity is just superficial. The radion has converted the nonlocal nonconventional Einstein equations to local quasi-scalar-tensor gravity.

We have also given a systematic method to calculate the corrections due to Kaluza-Klein massive modes. It is conjectured that all of the nonlocality arising from the integration is disentangled by the radion in the two-brane system. We have also emphasized the holographic aspect of our result. It turns out that the effect of bulk gravity on low energy physics in the brane world can be described solely in four-dimensional language. Conversely, the bulk geometry can be reconstructed from knowledge of the four-dimensional data. In this sense, the quasi-scalar-tensor gravity we have found in this paper works as a hologram and hence can be called holographic brane gravity.

Let us discuss some implications of our results. Cosmology is usually formulated on the basis of local field theory. However, superstring theory suggests that nonlocal field theories are ubiquitous. Although a nonlocal field theory is not easy to treat properly, the holographic description opens a new possibility for studying cosmology with nonlocal terms. Brane world cosmology can be regarded as a realization of a nonlocal field theoretic approach to cosmology. In the single brane picture, nonlocal terms due to the integration constant appear [12]. Furthermore, there are infinite series of higher derivative terms in the low energy expansion scheme. This is also a manifestation of the nonlocality of brane world gravity $[12,15]$. In the two-brane system, the above two types of nonlocality exist also. Intriguingly, the radion disentangles the nonlocality of the homogeneous solutions and leads to quasi-scalar-tensor gravity. Hence, the quasi-scalartensor theory is a nonlocal theory disguised as a local theory. In fact, integrating out the scalar field yields a nonlocal field theory. In addition, the nonlocality due to Kaluza-Klein type corrections remains as an infinite series in the low energy expansion even in the two-brane system. Cosmology with nonlocal fields from this point of view deserves further investigation.

As we have succeeded in obtaining the effective action for nonlinear brane gravity, various problems can now be investigated. Two-brane inflation is under investigation using our method [17]. Astrophysical applications such as gravitational waves from binary stars are also intriguing. Extension of our formalism to more general models that include bulk scalars or vector fields might be interesting.

\section{ACKNOWLEDGMENTS}

We would like to thank M. Sasaki for valuable suggestions which improved the presentation of the paper significantly. This work was supported in part by Monbukagakusho Grant-in-Aid No. 14540258.

\section{APPENDIX A: SCALAR FIELD EXAMPLE}

In order to illustrate the method used in the main text, we examine a toy model in this appendix. Let us consider a massless scalar field $\phi$ in the background

$$
d s^{2}=d y^{2}+\exp \left[-2 \frac{y}{l}\right] \eta_{\mu \nu} d x^{\mu} d x^{\nu},
$$

where the branes are located at $y=0$ and $y=d$. The equation of motion for $\phi$ with a source on the branes becomes

$$
\begin{aligned}
\square^{(5)} \phi & =e^{4 y / l} \partial_{y}\left[e^{-4 y / l} \partial_{y} \phi\right]+e^{2 y / l} \square \phi \\
& =J^{A}(x) \delta(y)+J^{B}(x) \delta(y-d) .
\end{aligned}
$$

From this equation, one can deduce the junction conditions

$$
\begin{aligned}
& \left.\partial_{y} \phi\right|_{y=0}=\frac{1}{2} J^{A}(x), \\
& \left.\partial_{y} \phi\right|_{y=d}=-\frac{1}{2} J^{B}(x) .
\end{aligned}
$$

Let us focus on the $A$-brane at $y=0$ and put

$$
\phi(y=0, x)=\phi_{0}(x) .
$$

The Green's function with the Neumann boundary condition is easily calculated as

$$
\begin{aligned}
\Delta_{5}\left(0, x ; 0, x^{\prime}\right) & =\int \frac{d^{4} p}{(2 \pi)^{4}} \exp \left[i p \cdot\left(x-x^{\prime}\right)\right] \frac{1}{q} \frac{J_{1}\left(q l e^{d / l}\right) H_{2}^{(1)}(q l)-J_{2}(q l) H_{1}^{(1)}\left(q l e^{d / l}\right)}{J_{1}\left(q l e^{d / l}\right) H_{1}^{(1)}(q l)-J_{1}(q l) H_{1}^{(1)}\left(q l e^{d / l}\right)} \\
& =\int \frac{d^{4} p}{(2 \pi)^{4}} \exp \left[i p \cdot\left(x-x^{\prime}\right)\right] \frac{2}{q^{2} l\left(1-e^{-2 d / l}\right)}\left[1+q^{2} l^{2}\left(\frac{3}{8}-\frac{1}{8} e^{-2 d / l}-\frac{1}{2\left(1-e^{-2 d / l}\right)} \frac{d}{l}\right)+\cdots\right],
\end{aligned}
$$

and 


$$
\begin{aligned}
\Delta_{5}\left(0, x ; d, x^{\prime}\right) & =-\frac{2 i e^{d / l}}{\pi l} \int \frac{d^{4} p}{(2 \pi)^{4}} \exp \left[i p \cdot\left(x-x^{\prime}\right)\right] \frac{1}{q^{2}} \frac{1}{J_{1}\left(q l e^{d / l}\right) H_{1}^{(1)}(q l)-J_{1}(q l) H_{1}^{(1)}\left(q l e^{d / l}\right)} \\
& =\int \frac{d^{4} p}{(2 \pi)^{4}} \exp \left[i p \cdot\left(x-x^{\prime}\right)\right] \frac{2}{q^{2} l\left(1-e^{-2 d / l}\right)}\left[1+q^{2} l^{2}\left(\frac{1}{8}+\frac{1}{8} e^{2 d / l}-\frac{1}{2\left(1-e^{-2 d / l}\right)} \frac{d}{l}\right)+\cdots\right]
\end{aligned}
$$

where $q^{2}=-\eta_{\mu \nu} p^{\mu} p^{\nu}$. Thus, the standard Green's function method gives the solution for Eq. (A2) as

$$
\phi_{0}(x)=\frac{1}{2} \int d^{4} x^{\prime} \Delta_{5}\left(0, x ; 0, x^{\prime}\right) J^{A}\left(x^{\prime}\right)+\frac{1}{2} \int d^{4} x^{\prime} e^{-4 d / l} \Delta_{5}\left(0, x ; d, x^{\prime}\right) J^{B}\left(x^{\prime}\right) .
$$

This gives

$$
\begin{aligned}
\square \phi_{0}(x)= & \frac{1}{2} \int d^{4} x^{\prime} \square \Delta_{5}\left(0, x ; 0, x^{\prime}\right) J^{A}\left(x^{\prime}\right)+\frac{1}{2} \int d^{4} x^{\prime} e^{-4 d / l} \square \Delta_{5}\left(0, x ; d, x^{\prime}\right) J^{B}\left(x^{\prime}\right) \\
= & \frac{1}{l\left(1-e^{-2 d / l}\right)} J^{A}(x)+\frac{e^{-4 d / l}}{l\left(1-e^{-2 d / l}\right)} e^{-4 d / l} J^{B}(x)+\frac{1}{l\left(1-e^{-2 d / l}\right)} \\
& \times\left[\frac{3}{8}-\frac{1}{8} e^{-2 d / l}-\frac{1}{2\left(1-e^{-2 d / l}\right)} \frac{d}{l}\right] l^{2} \square J^{A}(x)+\frac{e^{-4 d / l}}{l\left(1-e^{-2 d / l}\right)} e^{-4 d / l} \\
& \times\left[\frac{1}{8}+\frac{1}{8} e^{2 d / l}-\frac{1}{2\left(1-e^{-2 d / l}\right)} \frac{d}{l}\right] l^{2} \square J^{B}(x)+\cdots
\end{aligned}
$$

Note that the first two terms come from the zero mode and the rest are Kaluza-Klein corrections. Now we shall compare the above result Eq. (A9), with our method.

\section{Zeroth order}

At zeroth order, we ignore gradients on the brane; thus we get

$$
e^{4 y / l} \partial_{y}\left[e^{-4 y / l} \partial_{y} \phi^{(0)}\right]=0 .
$$

The solution of Eq. (A10) is given by

$$
\phi^{(0)}=\phi_{0}+e^{4 y / l} \psi_{0} .
$$

However, as we are regarding the source terms as first order quantities, the junction conditions (A3) and (A4) imply $\psi_{0}$ $=0$. Hence, we simply obtain $\phi^{(0)}=\phi_{0}$.

\section{First order}

At first order, we must solve

$$
e^{4 y / l} \partial_{y}\left[e^{-4 y / l} \partial_{y} \phi^{(1)}\right]=-e^{2 y / l} \square \phi_{0}(x) .
$$

The result is

$$
\phi^{(1)}=\frac{l^{2}}{4} e^{2 y / l} \square \phi_{0}+\frac{l}{4} e^{4 y / l} C(x)+D(x)
$$

where $C$ and $D$ are homogeneous solutions. The junction conditions (A3) and (A4) become

$$
\begin{aligned}
& \left.\partial_{y} \phi\right|_{y=0}=\frac{l}{2} \square \phi_{0}+C=\frac{1}{2} J^{A}(x), \\
& \left.\partial_{y} \phi\right|_{y=d}=\frac{l}{2} e^{2 d / l} \square \phi_{0}+C e^{4 d / l}=-\frac{1}{2} J^{B}(x) .
\end{aligned}
$$

Eliminating $C$ from these equations, we obtain

$$
\square \phi_{0}=\frac{1}{l\left(1-e^{-2 d / l}\right)} J^{A}+\frac{e^{-4 d / l}}{l\left(1-e^{-2 d / l}\right)} J^{B} .
$$

This agrees with the zero mode part of Eq. (A9). Thus our method to first order corresponds to the zero mode truncation when linearized. The homogeneous part is also determined as

$$
C(x)=\frac{1}{2\left(1-e^{2 d / l}\right)}\left[J^{A}+e^{-2 d / l} J^{B}\right] .
$$

\section{Second order}

At the second order, we have

$$
e^{4 y / l} \partial_{y}\left[e^{-4 y / l} \partial_{y} \phi^{(2)}\right]=-e^{2 y / l} \square \phi^{(1)}(x) .
$$

Equation (A18) can be integrated as 


$$
\begin{aligned}
\partial_{y} \phi^{(2)}= & -\frac{l^{2}}{4}\left(y e^{4 y / l}+\frac{l}{2} e^{2 y / l}\right) \square^{2} \phi_{0} \\
& -\frac{l^{2}}{8}\left(e^{6 y / l}+e^{2 y / l}\right) \square C(x)+e^{4 y / l} D(x) .
\end{aligned}
$$

Hence, the junction conditions (A3) and (A4) yield

$$
\begin{gathered}
\frac{l}{2} \square \phi_{0}+C(x)-\frac{l^{3}}{8} \square^{2} \phi_{0}-\frac{l^{2}}{4} \square C(x)+D(x)=\frac{1}{2} J^{A}(x), \\
\frac{l}{2} e^{2 y / l} \square \phi_{0}+C(x) e^{4 y / l}-\frac{l^{2}}{4}\left(d e^{4 y / l}+\frac{l}{2} e^{2 y / l}\right) \square^{2} \phi_{0} \\
-\frac{l^{2}}{8}\left(e^{6 y / l}+e^{2 y / l}\right) \square C(x)+e^{4 y / l} D(x)=-\frac{1}{2} J^{B}(x) .
\end{gathered}
$$

Combining both Eqs. (A20) and (A21), we get

$$
\begin{aligned}
\square \phi_{0}= & \frac{1}{l\left(1-e^{-2 d / l}\right)}\left[J^{A}+e^{-4 d / l} J^{B}\right] \\
& +\left[\frac{l^{2}}{4}-\frac{l d}{2} \frac{1}{\left(1-e^{-2 d / l}\right)}\right] \square^{2} \phi_{0} \\
& -\frac{l}{4} e^{2 d / l}\left[1-e^{-2 d / l}\right] \square C .
\end{aligned}
$$

Substituting Eqs. (A16) and (A17) into the right-hand side of Eq. (A22) yields Eq. (A9). Thus, we have shown that the second order equations in our method correspond to taking into account the Kaluza-Klein corrections when the equations are linearized.

\section{APPENDIX B: LINEARIZED GRAVITY}

Let us now turn to our case of interest, that is, linearized gravity. In linearized gravity, following the method in [5], the solution is explicitly given in terms of the scalar Neumann Green's function $\Delta_{5}$ in Eqs. (A6) and (A7):

$$
\begin{aligned}
\bar{h}_{\mu \nu}^{A}\left(x^{\mu}\right)= & -\kappa^{2} \int d^{4} x^{\prime} \Delta_{5}\left(0, x^{\mu} ; 0, x^{\mu^{\prime}}\right)\left[T_{\mu \nu}^{A}\left(x^{\prime}\right)-\frac{1}{6} \eta_{\mu \nu} T^{A}\left(x^{\prime}\right)\right]-\frac{1}{3} \frac{\kappa^{2}}{l} \eta_{\mu \nu} \int d^{4} x^{\prime} \Delta_{4}\left(x^{\mu}, x^{\mu^{\prime}}\right) T^{A}\left(x^{\prime}\right) \\
& -\kappa^{2} \int d^{4} x^{\prime} e^{-2 d / l} \Delta_{5}\left(0, x^{\mu} ; d, x^{\mu^{\prime}}\right)\left[T_{\mu \nu}^{B}\left(x^{\prime}\right)-\frac{1}{6} \eta_{\mu \nu} T^{B}\left(x^{\prime}\right)\right]
\end{aligned}
$$

where $\bar{h}_{\mu \nu}^{A}$ is the small fluctuation in the metric on the $A$-brane. Applying $\square$ to this equation and expanding $\Delta_{5}$ as Eqs. (A6) and (A7), we obtain

$$
\begin{aligned}
\square \bar{h}_{\mu \nu}^{A}= & -\frac{2 \kappa^{2}}{l} \frac{1}{1-e^{-2 d / l}}\left(\begin{array}{c}
{ }^{A} \\
T_{\mu \nu}+e^{-2 d / l} T_{\mu \nu}^{B}
\end{array}\right)+\frac{1}{3} \frac{\kappa^{2}}{l} \frac{e^{-2 d / l}}{1-e^{-2 d / l}} \eta_{\mu \nu}\left(\begin{array}{c}
A \\
T+T
\end{array}\right)-2 \frac{\kappa^{2}}{l} \frac{1}{1-e^{-2 d / l}[}\left[\frac{3}{8}-\frac{1}{8} e^{-2 d / l}\right. \\
& \left.-\frac{d / l}{2\left(1-e^{-2 d / l}\right)}\right] l^{2} \square\left(\begin{array}{c}
A \\
T_{\mu \nu}-\frac{1}{6} \eta_{\mu \nu} T
\end{array}\right)-2 \frac{\kappa^{2}}{l} \frac{e^{-2 d / l}}{1-e^{-2 d / l}}\left[\frac{1}{8}+\frac{1}{8} e^{2 d / l}-\frac{d / l}{2\left(1-e^{-2 d / l}\right)}\right] l^{2} \square\left(\begin{array}{c}
B \\
T_{\mu \nu}-\frac{1}{6} \eta_{\mu \nu} T
\end{array}\right) .
\end{aligned}
$$

This may be regarded as the effective Einstein equation corrected to $O\left((l / L)^{4}\right)$. Now we demonstrate that our low energy expansion scheme leads to linearized quasi-Brans-Dicke gravity. Then we will show that our method correctly reproduces Eq. (B2).

Our solution for the bulk metric is

$$
d s^{2}=e^{2 \phi(y, x)} d y^{2}+\exp \left[-\frac{2}{l} \int d y e^{\phi(y, x)}\right] h_{\mu \nu}\left(x^{\mu}\right) d x^{\mu} d x^{\nu} .
$$

Here, two branes are located at $y=0$ and $y=l$. We will assume that $\phi\left(y, x^{\mu}\right) \equiv \phi\left(x^{\mu}\right)$ for simplicity. After some obvious changes of variables and rescalings of coordinates, small fluctuations in the metric can be represented as

$$
\begin{aligned}
d s^{2}= & (1+2 \delta \phi) d y^{2} \\
& +e^{-(2 / l) y}\left(\eta_{\mu \nu}+h_{\mu \nu}\left(x^{\mu}\right)-\frac{2 y}{l} \eta_{\mu \nu} \delta \phi\left(x^{\mu}\right)\right) d x^{\mu} d x^{\nu} ;
\end{aligned}
$$

thus

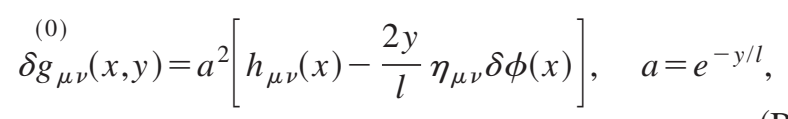

where $h_{\mu \nu}$ and $\delta \phi$ represent tensor and scalar fluctuations, respectively. Now the two branes are located at $y=0$ and $y$ $=d$, because of the relation $e^{\phi}=d / l$. Decomposing the ex- 
trinsic curvature into the traceless part and the trace part, the small fluctuations of each part are

$$
\Sigma_{\nu}^{\mu}=\delta \Sigma_{\nu}^{\mu}, \quad Q=\frac{4}{l}+\delta Q
$$

Here we used our results in Eq. (30). The equations off the brane, Eqs. (12)-(15), are linearized to become

$$
\begin{aligned}
& \delta \Sigma_{\nu, y}^{\mu}-\frac{4}{l} \delta \Sigma_{\nu}^{\mu}=-\left[R_{\nu}^{\mu}-\frac{1}{4} \delta_{\nu}^{\mu} R-\nabla^{\mu} \nabla_{\nu} \delta \phi\right. \\
&\left.+\frac{1}{4} \delta_{\nu}^{\mu} \nabla^{\alpha} \nabla_{\alpha} \delta \phi\right], \\
& \delta Q= \frac{l}{6}[R], \\
& \delta Q_{, y}-\frac{2}{l} \delta Q=\nabla^{\alpha} \nabla_{\alpha} \delta \phi, \\
& \nabla_{\lambda} \delta \Sigma_{\mu}^{\lambda}-\frac{3}{4} \nabla_{\mu} \delta Q=0 .
\end{aligned}
$$

The junction conditions become

$$
\begin{gathered}
{\left.\left[\delta \Sigma_{\nu}^{\mu}{ }_{\nu}-\frac{3}{4} \delta_{\nu}^{\mu} \delta Q\right]\right|_{y=0}=\frac{\kappa^{2}}{2} T^{\mu}{ }_{\nu},} \\
{\left.\left[\delta \Sigma_{\nu}^{\mu}-\frac{3}{4} \delta_{\nu}^{\mu} \delta Q\right]\right|_{y=d}=-\frac{\kappa^{2}}{2} \widetilde{T}_{\nu}^{\mu} .}
\end{gathered}
$$

We now work with our low energy iteration scheme. The goal is to construct the metric fluctuation as

$$
\begin{aligned}
\delta g_{\mu \nu}(x, y)= & a^{2}\left[h_{\mu \nu}(x)-\frac{2 y}{l} \eta_{\mu \nu} \delta \phi(x)+\delta g_{\mu \nu}^{(1)}(x, y)\right. \\
& \left.+\delta g_{\mu \nu}(x, y)+\cdots\right] .
\end{aligned}
$$

\section{First order}

The solution at the first order is

$$
\begin{aligned}
\stackrel{(1)}{\delta Q}= & \frac{l}{6 a^{2}} R(h)+\frac{y}{a^{2}} \square \delta \phi, \\
\delta \Sigma_{\nu}^{\mu}= & \frac{l}{2 a^{2}}\left(R_{\nu}^{\mu}-\frac{1}{4} \delta_{\nu}^{\mu} R\right)+\frac{y}{a^{2}}\left(\delta \phi^{\mid \mu}{ }_{\mid \nu}-\frac{1}{4} \delta_{\nu}^{\mu} \square \delta \phi\right) \\
& +\frac{\chi^{\mu}{ }_{\nu}}{a^{4}}, \quad \chi_{\mu}^{\mu}=0,
\end{aligned}
$$

$$
\begin{aligned}
\delta K_{\nu}^{\mu}= & \frac{l}{2 a^{2}}\left(R_{\nu}^{\mu}-\frac{1}{6} \delta^{\mu}{ }_{\nu} R\right)+\frac{y}{a^{2}} \delta \phi_{\mid \nu}^{\mid \mu}+\frac{\chi_{\nu}^{\mu}}{a^{4}}, \\
\delta g_{\mu \nu}^{(1)}= & -\frac{l^{2}}{2}\left(\frac{1}{a^{2}}-1\right)\left(R_{\mu \nu}-\frac{1}{6} \eta_{\mu \nu} R\right) \\
& -\left(\frac{l y}{a^{2}}-\frac{l^{2}}{2 a^{2}}+\frac{l^{2}}{2}\right) \delta \phi_{\mid \mu \nu}-\frac{l}{2}\left(\frac{1}{a^{4}}-1\right) \chi_{\mu \nu} .
\end{aligned}
$$

From Eq. (B10), we obtain the constraint $\chi_{\nu \mid \mu}^{\mu}=0$ for the homogeneous solution. The junction conditions are

$$
\begin{aligned}
& {\left.\left[\delta \Sigma^{(1)}{ }_{\nu}-\frac{3}{4} \delta_{\nu}^{\mu} \delta Q\right]\right|_{y=0}=\frac{l}{2} G_{\nu}^{\mu}+\chi_{\nu}^{\mu}=\frac{\kappa^{2}{ }^{A}}{2} T_{\nu}^{\mu},} \\
& {\left.\left[\delta \Sigma_{\nu}^{\mu}-\frac{3}{4} \delta_{\nu}^{\mu} \delta Q\right]\right|_{y=d}=\frac{l}{2 \Omega^{2}} G_{\nu}^{\mu}+\frac{l}{\Omega^{2}}\left(\delta \phi^{\mid \mu}{ }_{\mid \nu}\right.} \\
& \left.-\delta_{\nu}^{\mu} \square \delta \phi\right)+\frac{\chi^{\mu}{ }_{\nu}}{\Omega^{4}} \\
& =-\frac{\kappa^{2}}{2 \Omega^{2}} T_{\nu}^{\mu} .
\end{aligned}
$$

Here, we used the relation (49) between $\widetilde{T}^{\mu}{ }_{\nu}$ and $T^{\beta}{ }_{\nu}$. The homogeneous solution $\chi^{\mu}{ }_{\nu}$ can be eliminated from Eqs. (B18) and (B19) to yield

$$
\begin{aligned}
& G_{\nu}^{\mu}=\frac{\kappa^{2}}{l} \frac{1}{1-\Omega^{2}}\left(\begin{array}{c}
A \\
T_{\nu}^{\mu} \\
\nu
\end{array} \Omega^{2} T_{\nu}^{\mu}{ }_{\nu}\right)+\frac{2 \Omega^{2}}{1-\Omega^{2}}\left(\delta \phi^{\mid \mu}{ }_{\nu}\right. \\
& \left.-\delta_{\nu}^{\mu} \square \delta \phi\right) \text {. }
\end{aligned}
$$

We now introduce a linearized version of the field $\Psi$ introduced in Eq. (51) by $\Psi=\left(1-\Omega^{2}\right)+2 \Omega^{2} \delta \phi \equiv \Psi_{0}+\delta \Psi$. The linearized effective equations can then be written as

$$
G_{\nu}^{\mu}=\frac{\kappa^{2}}{l \Psi_{0}}\left(\begin{array}{c}
{ }^{A} \\
T^{\mu}{ }_{\nu}+\left(1-\Psi_{0}\right) T_{\nu}^{\mu}
\end{array}\right)+\frac{1}{\Psi_{0}}\left(\delta \Psi_{\mid \nu}^{\mid \mu}-\delta_{\nu}^{\mu} \square \delta \Psi\right) .
$$

Equations (B11) and (B12) determine the homogeneous solution $\chi^{\mu}{ }_{\nu}$ as

$$
\chi_{\nu}^{\mu}=-\frac{\kappa^{2}}{2} \frac{1-\Psi_{0}}{\Psi_{0}}\left(\begin{array}{c}
{ }^{A} \\
T_{\nu}^{\mu} \\
\nu
\end{array} T^{B}{ }_{\nu}\right)-\frac{l}{2 \Psi_{0}}\left(\delta \Psi_{\mid \nu}^{\mid \mu}-\delta_{\nu}^{\mu} \square \delta \Psi\right) .
$$

The traceless condition of $\chi_{\nu}^{\mu}$ leads to 


$$
\square \delta \Psi=\frac{\kappa^{2}}{l} \frac{\begin{array}{c}
A \quad B \\
2 \omega+3
\end{array}}{2 .}
$$

Thus we found that linearizing our method leads to "linearized quasi-Brans-Dicke gravity" with the Brans-Dicke parameter

$$
\omega=\frac{3}{2}\left(\frac{1}{\Omega^{2}}-1\right)=\frac{3}{2}\left(e^{2 d / l}-1\right) .
$$

By linearizing $G_{\nu}^{\mu}$ in Eq. (B20) and defining

$$
\bar{h}_{\mu \nu}=h_{\mu \nu}-\frac{1}{2} \eta_{\mu \nu} h
$$

one gets

$$
\begin{aligned}
\frac{1}{2}\left(\bar{h}_{\mid \nu \alpha}^{\alpha \mu}+\bar{h}_{\nu}^{\alpha \mid \mu}{ }_{\nu}-\square \bar{h}_{\nu}^{\mu}{ }_{\nu}-\delta_{\nu}^{\mu} \bar{h}^{\alpha \beta}{ }_{\mid \alpha \beta}\right) \\
=\frac{2 \Omega^{2}}{1-\Omega^{2}}\left(\delta \phi^{\mid \mu}{ }_{\mid \nu}-\delta_{\nu}^{\mu} \square \delta \phi\right)+\frac{\kappa^{2}}{l} \frac{1}{1-\Omega^{2}} \\
\quad \times\left(\begin{array}{c}
A \\
T_{\nu}^{\mu}+\Omega^{2} T^{\mu}{ }_{\nu}
\end{array}\right) .
\end{aligned}
$$

Note that $h^{\mu}{ }_{\nu}(x)$ is the fluctuation of the induced metric on the $A$-brane located at $y=0$. The gauge freedom can be used to set

$$
\bar{h}_{\mid \alpha}^{\alpha \mu}=\frac{2 \Omega^{2}}{1-\Omega^{2}} \delta \phi^{\mid \mu}
$$

and then Eq. (B26) becomes

$$
\begin{aligned}
\square \bar{h}_{\nu}^{\mu}= & -\frac{2 \kappa^{2}}{l} \frac{1}{1-\Omega^{2}}\left(\begin{array}{c}
A \\
T_{\nu}^{\mu}+\Omega^{2} T_{\nu}{ }_{\nu}
\end{array}\right) \\
& +\frac{\kappa^{2}}{3 l} \frac{\Omega^{2}}{1-\Omega^{2}} \delta_{\nu}^{\mu}\left(\begin{array}{cr}
A & B \\
T+T
\end{array}\right) .
\end{aligned}
$$

This is in agreement with the leading order term in Eq. (B2) and of course is the same as the one derived by Garriga and Tanaka [6].

\section{Second order}

Next we compute the second order solution. The basic equations become

$$
\begin{gathered}
\delta \Sigma_{\nu, y}^{\mu}-\frac{4}{l} \delta \Sigma_{\nu}^{\mu}{ }_{\nu}^{(2)}=-\left[R_{\nu}^{\mu}-\frac{1}{4} \delta_{\nu}^{\mu} R\right]^{(2)}, \\
\delta Q=\frac{l}{6}[R]^{(2)}
\end{gathered}
$$

$$
\begin{aligned}
& \delta Q_{, y}-\frac{2}{l} \delta Q=0 \text {, } \\
& \nabla_{\lambda} \delta \Sigma_{\mu}^{\lambda}-\frac{3}{4} \nabla_{\mu} \delta Q=0 .
\end{aligned}
$$

The junction conditions are

$$
\left.\left[\delta \Sigma_{\nu}^{\mu}-\frac{3}{4} \delta_{\nu}^{\mu} \delta Q\right]\right|_{y=0}=\frac{\kappa^{2 A(2)}}{2} T_{\nu}^{\mu}
$$

$$
\left.\left[\delta \Sigma^{\mu}{ }_{\nu}-\frac{3}{4} \delta_{\nu}^{\mu} \delta Q\right]\right|_{y=d}=-\frac{\kappa^{2}{ }^{B(2)}}{2 \Omega^{2}} T^{\mu}{ }_{\nu} .
$$

From Eqs. (B29) and (B30), the solution is

$$
\delta Q=0
$$

and

$$
\begin{aligned}
\delta \Sigma_{\nu}^{\mu}{ }_{\nu} & =\frac{l^{2}}{4}\left(\frac{y}{a^{4}}+\frac{l}{2 a^{2}}\right) \mathcal{S}_{\nu}^{\mu}-\frac{l^{2}}{8}\left(\frac{1}{a^{6}}+\frac{1}{a^{2}}\right) \square \chi_{\nu}^{\mu}+\frac{C^{\mu}{ }_{\nu}}{a^{4}}, \\
C^{\mu}{ }_{\mu} & =0 .
\end{aligned}
$$

Here we have introduced the tensor $\mathcal{S}_{\nu}^{\mu}$,

$$
\mathcal{S}^{\mu}{ }_{\nu}=\frac{1}{3} R^{\mid \mu}{ }_{\mid \nu}-\square R_{\nu}^{\mu}+\frac{1}{6} \delta^{\mu}{ }_{\nu} \square R,
$$

with the properties $\mathcal{S}^{\mu}{ }_{\nu \mid \mu}=\mathcal{S}_{\mu}^{\mu}=0$.

Equation (B31) is trivially satisfied by $\delta Q$ in Eq. (B35). To satisfy Eq. (B32), the homogeneous solution in $\delta \Sigma_{\nu}^{\mu}$ is constrained as $C_{\nu \mid \mu}^{\mu}=0$. The junction conditions (B33) and (B34) then give

$$
\begin{gathered}
\frac{l^{3}}{8} \mathcal{S}^{\mu}{ }_{\nu}-\frac{l^{2}}{4} \square \chi^{\mu}{ }_{\nu}+C^{\mu}{ }_{\nu}=\frac{\kappa^{2}}{2} T^{A(2)}{ }_{\nu}, \\
\frac{l^{2}}{4}\left(\frac{d}{\Omega^{4}}+\frac{l}{2 \Omega^{2}}\right) \mathcal{S}_{\nu}^{\mu}-\frac{l^{2}}{8}\left(\frac{1}{\Omega^{6}}+\frac{1}{\Omega^{2}}\right) \square \chi_{\nu}^{\mu}{ }_{\nu} \\
+\frac{C^{\mu}{ }_{\nu}}{\Omega^{4}}=-\frac{\kappa^{2}}{2 \Omega^{2}} T_{\nu}^{\mu(2)}{ }_{\nu} .
\end{gathered}
$$

By combining Eqs. (B38) and (B39) with the junction conditions at first order, we obtain the following equations:

$$
\frac{l}{2} G_{\nu}^{\mu}+\chi^{\mu}{ }_{\nu}+\frac{l^{3}}{8} \mathcal{S}^{\mu}{ }_{\nu}-\frac{l^{2}}{4} \square \chi^{\mu}{ }_{\nu}+C^{\mu}{ }_{\nu}=\frac{\kappa^{2}}{2} T^{A}{ }_{\nu},
$$




$$
\begin{gathered}
\frac{l}{2} G_{\nu}^{\mu}+\frac{\chi^{\mu}{ }_{\nu}}{\Omega^{2}}+\frac{l^{2}}{4}\left(\frac{d}{\Omega^{2}}+\frac{l}{2}\right) \mathcal{S}_{\nu}^{\mu}-\frac{l^{2}}{8}\left(\frac{1}{\Omega^{4}}+1\right) \square \chi_{\nu}^{\mu} \\
+l\left(\delta \phi^{\mid \mu}{ }_{\mid \nu}-\delta_{\nu}^{\mu} \square \delta \phi\right)+\frac{C^{\mu}{ }_{\nu}}{\Omega^{2}}=-\frac{\kappa^{2}}{2} T^{B}{ }_{\nu} .
\end{gathered}
$$

Eliminating $\mathrm{C}^{\mu}{ }_{\nu}$ from the above two equations, we obtain the effective four-dimensional theory of gravity with correction terms:

$$
\begin{aligned}
G_{\nu}^{\mu}= & \frac{\kappa^{2}}{l} \frac{1}{1-\Omega^{2}}\left(T_{\nu}^{A}+\Omega^{2} T^{\mu}{ }_{\nu}\right)+\frac{2 \Omega^{2}}{1-\Omega^{2}}\left(\delta \phi^{\mid \mu}{ }_{\nu}\right. \\
& \left.-\delta_{\nu}^{\mu} \square \delta \phi\right)+\frac{l^{2}}{2\left(1-\Omega^{2}\right)}\left(\frac{d}{l}-\frac{1-\Omega^{2}}{2}\right) \mathcal{S}^{\mu}{ }_{\nu} \\
& -\frac{l}{4} \frac{1-\Omega^{2}}{\Omega^{2}} \square \chi^{\mu}{ }_{\nu} .
\end{aligned}
$$

If we rewrite this equation using $\Psi$, we get

$$
\begin{aligned}
G_{\nu}^{\mu}= & \frac{\kappa^{2}}{l \Psi_{0}}\left(\begin{array}{c}
{ }^{A} \\
T^{\mu}{ }_{\nu}+\left(1-\Psi_{0}\right) T^{\mu}{ }_{\nu}
\end{array}\right)+\frac{1}{\Psi_{0}}\left(\delta \Psi^{\mid \mu}{ }_{\mid \nu}-\delta_{\nu}^{\mu} \square \delta \Psi\right) \\
& +\frac{l^{2}}{2 \Psi_{0}}\left(\frac{d}{l}-\frac{\Psi_{0}}{2}\right) \mathcal{S}^{\mu}{ }_{\nu}-\frac{l}{4} \frac{\Psi_{0}}{1-\Psi_{0}} \square \chi^{\mu}{ }_{\nu} .
\end{aligned}
$$

Note that $\chi_{\nu}^{\mu}$, given by the traceless part of Eq. (B22), satisfies the transverse-traceless condition. Thus we have obtained the linearized quasi-Brans-Dicke theory with KaluzaKlein corrections. Using $\bar{h}_{\mu \nu}$ defined by Eq. (B25), Eq. (B42) leads to

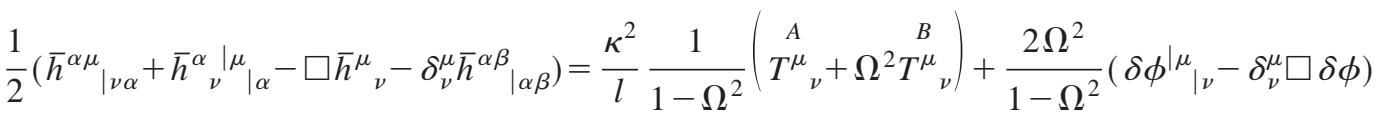

$$
\begin{aligned}
& +\frac{l^{2}}{2\left(1-\Omega^{2}\right)}\left(\frac{d}{l}-\frac{1-\Omega^{2}}{2}\right)\left(\frac{1}{3} \bar{h}_{\mid \alpha \beta}^{\alpha \beta}{ }_{\mid \nu}^{\mid \mu}+\frac{1}{6} \square \bar{h}_{\mid \nu}^{\mid \mu}-\frac{1}{2} \square \bar{h}_{\mid \nu \alpha}^{\mu \alpha}-\frac{1}{2} \square \bar{h}_{\nu}^{\alpha \mid \mu}{ }_{\nu}\right. \\
& \left.+\frac{1}{2} \square^{2} \bar{h}_{\nu}^{\mu}+\frac{1}{6} \delta_{\nu}^{\mu} \square \bar{h}_{\alpha \beta} \mid \alpha \beta-\frac{1}{6} \delta_{\nu}^{\mu} \square^{2} \bar{h}\right) \\
& +\frac{l}{4} \frac{1-\Omega^{2}}{\Omega^{2}}\left[\frac{l \Omega^{2}}{1-\Omega^{2}}\left(\square \delta \phi_{\mid \nu}^{\mid \mu}-\delta_{\nu}^{\mu} \square^{2} \delta \phi\right)-\frac{\kappa^{2}}{2} \frac{\Omega^{2}}{1-\Omega^{2}} \square\left(T_{\nu}^{\mu}{ }_{\nu}+T_{\nu}^{\mu}\right)\right] .
\end{aligned}
$$

Imposing the gauge condition

$$
\bar{h}_{\mid \alpha}^{\alpha \mu}=\frac{2 \Omega^{2}}{1-\Omega^{2}} \delta \phi^{\mid \mu}-\left[\frac{4 l^{2}}{3} \frac{\Omega^{2}}{1-\Omega^{2}}\left(\frac{d / l}{2\left(1-\Omega^{2}\right)}-\frac{1}{4}\right)-\frac{l^{2}}{4}\right] \square \delta \phi^{\mid \mu}+\frac{l^{2}}{6}\left[\frac{d / l}{2\left(1-\Omega^{2}\right)}-\frac{1}{4}\right] \square \bar{h}^{\mid \mu},
$$

we get the following equation:

$$
\begin{aligned}
& \square \bar{h}^{\mu}{ }_{\nu}=-\frac{2 \kappa^{2}}{l} \frac{1}{1-\Omega^{2}}\left(\begin{array}{c}
{ }_{A} \\
T^{\mu}{ }_{\nu}+\Omega^{2} T^{\mu}{ }_{\nu}
\end{array}\right)+\frac{\kappa^{2}}{3 l} \frac{\Omega^{2}}{1-\Omega^{2}} \delta_{\nu}^{\mu}\left(\begin{array}{c}
A \\
T+T
\end{array}\right)-\frac{2 l \kappa^{2}}{1-\Omega^{2}}\left[\frac{3}{8}-\frac{1}{8} \Omega^{2}-\frac{d / l}{2\left(1-\Omega^{2}\right)}\right] \square\left(\begin{array}{c}
A \\
T^{\mu}{ }_{\nu}-\frac{1}{6}{ }^{A}{ }_{\nu}^{\mu} T
\end{array}\right) \\
& -\frac{2 l \kappa^{2} \Omega^{2}}{1-\Omega^{2}}\left[\frac{1}{8}+\frac{1}{8} \frac{1}{\Omega^{2}}-\frac{d / l}{2\left(1-\Omega^{2}\right)}\right] \square\left(\begin{array}{c}
B \\
T_{\nu}^{\mu}
\end{array}-\frac{1}{6} \delta_{\nu}^{\mu} T\right) .
\end{aligned}
$$

This result coincides with the result of the standard linear theory given in Eq. (B2). 
[1] L. Randall and R. Sundrum, Phys. Rev. Lett. 83, 4690 (1999); 83, 3370 (1999).

[2] N. Kaloper, Phys. Rev. D 60, 123506 (1999); K. Koyama and J. Soda, Phys. Lett. B 483, 432 (2000); S. Kobayashi, K. Koyama, and J. Soda, Phys. Rev. D 65, 064014 (2002); H.A. Chamblin and H.S. Reall, Nucl. Phys. B562, 133 (1999).

[3] P. Kraus, J. High Energy Phys. 12, 011 (1999); S. Mukohyama, Phys. Lett. B 473, 241 (2000); P. Binétruy, C. Deffayet, U. Ellwanger, and D. Langlois, ibid. 477, 285 (2000); D. Ida, J. High Energy Phys. 09, 014 (2000); E. Flanagan, S. Tye, and I. Wasserman, Phys. Rev. D 62, 024011 (2000).

[4] K. Koyama and J. Soda, Phys. Rev. D 62, 123502 (2000); S. Mukohyama, ibid. 62, 084015 (2000); H. Kodama, A. Ishibashi, and O. Seto, ibid. 62, 064022 (2000); C. van de Bruck, M.

Dorca, R.H. Brandenberger, and A. Lukas, ibid. 62, 123515 (2000); D. Langlois, ibid. 62, 126012 (2000); R. Maartens, ibid. 62, 084023 (2000); N. Deruelle, T. Dolezel, and J. Katz, ibid. 63, 083513 (2001); K. Koyama and J. Soda, ibid. 65, 023514 (2002); D. Langlois, R. Maartens, M. Sasaki, and D. Wands, ibid. 63, 084009 (2001); B. Leong, P. Dunsby, A. Challinor, and A. Lasenby, ibid. 65, 104012 (2002).

[5] S. Giddings, E. Katz, and L. Randall, J. High Energy Phys. 03, 023 (2000).

[6] J. Garriga and T. Tanaka, Phys. Rev. Lett. 84, 2778 (2000).

[7] C. Charmousis, R. Gregory, and V.A. Rubakov, Phys. Rev. D 62, 067505 (2000).
[8] U. Gen and M. Sasaki, Prog. Theor. Phys. 105, 591 (2001).

[9] P. Binetruy, C. Deffayet, and D. Langlois, Nucl. Phys. B615, 219 (2001); T. Chiba, Phys. Rev. D 62, 021502(R) (2000); Z. Chacko and P. Fox, ibid. 64, 024015 (2001); D. Langlois and L. Sorbo, hep-th/0203036; A. Das and A. Mitov, Phys. Rev. D 66, 045030 (2002); H. Kudoh and T. Tanaka, ibid. 65, 104034 (2002); A.O. Barvinsky, ibid. 65, 062003 (2002).

[10] K. Koyama, Phys. Rev. D (to be published), gr-qc/0204047; J.M. Cline and H. Firouzjahi, Phys. Lett. B 495, 271 (2000); U. Ellwanger, hep-th/0001126.

[11] T. Wiseman, Class. Quantum Grav. 19, 3083 (2002).

[12] S. Kanno and J. Soda, Phys. Rev. D 66, 043526 (2002).

[13] T. Shiromizu, K. Maeda, and M. Sasaki, Phys. Rev. D 62, 024012 (2000).

[14] K. Tomita, Prog. Theor. Phys. 54, 730 (1975); G.L. Comer, N. Deruelle, D. Langlois, and J. Parry, Phys. Rev. D 49, 2759 (1994); D.S. Salopek and J.M. Stewart, ibid. 47, 3235 (1993); J. Soda, H. Ishihara, and O. Iguchi, Prog. Theor. Phys. 94, 781 (1995).

[15] S. Mukohyama, Phys. Rev. D 64, 064006 (2001); 65, 084036 (2002).

[16] N. Deruelle and J. Katz, Phys. Rev. D 64, 083515 (2001); T. Wiseman, ibid. 65, 124007 (2002); R. Emparan, A. Fabbri, and N. Kaloper, hep-th/0206155.

[17] S. Kanno and J. Soda (work in progress). 